\title{
Les événements de Ghardaïa et l'usage des réseaux sociaux numériques en Algérie
}

De la mobilisation sociale à l'intelligence collective

The events of Ghardaia and the use of digital social networks in Algeria : social mobilization for collective intelligence

Los acontecimientos de Ghardaia y el uso de las redes sociales digitales en Argelia : la movilización social para la inteligencia colectiva

\section{Rima Rouibi}

\section{OpenEdition}

\section{Journals}

Édition électronique

URL : http://journals.openedition.org/ctd/969

DOI : $10.4000 /$ ctd. 969

ISSN : 2491-1437

Éditeur

Chaire Unesco Pratiques émergentes en technologies et communication pour le développement

Édition imprimée

Date de publication : 30 octobre 2016

ISBN : 2491-1437

Référence électronique

Rima Rouibi, «Les événements de Ghardaïa et l'usage des réseaux sociaux numériques en Algérie », Communication, technologies et développement [En ligne], 3 | 2016, mis en ligne le 30 octobre 2016, consulté le 13 juin 2020. URL : http://journals.openedition.org/ctd/969 ; DOI : https://doi.org/10.4000/ ctd.969

Ce document a été généré automatiquement le 13 juin 2020.

Communication, technologies et développement 


\section{Les événements de Ghardaïa et l'usage des réseaux sociaux numériques en Algérie}

De la mobilisation sociale à l'intelligence collective

The events of Ghardaia and the use of digital social networks in Algeria : social mobilization for collective intelligence

Los acontecimientos de Ghardaia y el uso de las redes sociales digitales en Argelia : la movilización social para la inteligencia colectiva

\section{Rima Rouibi}

\section{Introduction}

1 En Algérie, il n'y a pas eu de «révolution » semblable à celles de Tunisie (14 janvier 2011) ou d'Égypte (25 janvier 2011). Seules quelques émeutes contre la cherté de la vie, en janvier 2011, et des mouvements de protestations sectorielles ont ponctué la vie politique du pays. La réponse du gouvernement a été de promettre une série de réformes annoncées par le président Bouteflika dans son discours d'avril 2011. Mais il a fallu attendre cinq ans pour que ces réformes aboutissent avec l'adoption des amendements constitutionnels le 7 février $2016^{1}$.

Durant ces cinq dernières années, des émeutes ont eu lieu dans la région de Ghardaïa, au sud du pays, avec pour toile de fond des questions d'ordre ethnique et/ou religieux. Ces clivages, qui ont fait émerger les questions communautaires dans le discours médiatique en Algérie, ont été exploités par les tenants de la polarisation dans chacun des deux camps, Chaambas² Malékites (Arabes sunnites) et Mozabites Ibadites (Berbères de doctrine kharédjite).

3 Le présent article se propose de jeter la lumière sur l'usage des réseaux sociaux numériques par les acteurs de la crise de Ghardaïa et de comprendre les ressorts du discours utilisé tantôt pour attiser l'affrontement, tantôt dans une posture plus 
conciliante. Ici, une question mérite d'être posée: dans quelle mesure les réseaux sociaux numériques ont-ils contribué à l'exacerbation des haines entre les protagonistes (Malékites et Ibadites), avant d'œuvrer à l'apaisement dans la région de Ghardaïa?

Pour ce faire, ce travail de recherche mobilise deux notions clés: les mouvements sociaux et l'intelligence collective. La première peut être définie comme la signification la plus élevée d'une action collective à la fois contestataire et défensive, et visant au contrôle de l'historicité, tandis que la seconde tend à comprendre de manière de plus en plus précise et opératoire le fonctionnement des groupes humains engagés dans une activité coopérative au moyen d'ordinateurs - ou de terminaux mobiles - en réseaux. Ces notions seront développées plus loin dans l'analyse. Notre réflexion initiale se focalise sur l'usage des réseaux sociaux numériques par certains protagonistes de la crise à Ghardaïa, et le rôle de ces derniers dans l'évolution de cette crise.

Bien que les émeutes cycliques à Ghardaïa remontent à 1985, cette étude de cas, consacrée à celles de 2014-2015, a pour objectif d'éclairer une pratique encore peu répandue en Algérie, à savoir la mobilisation sociale et politique via les réseaux sociaux numériques. En effet, la crise de Ghardaïa a confirmé, s'il en est, cette nouvelle conscience sociale quant aux possibilités que présente l'utilisation de YouTube et de Facebook comme vecteur à même d'influencer la vie de la collectivité nationale.

Nous observerons cependant un usage différencié et hiérarchisé de ces réseaux de la part des représentants se réclamant de chaque partie (Malékites et Ibadites). Les premiers, représentés par le jeune activiste Ahmed Seklab, qui s'est imposé au fil des événements comme un représentant de la partie chaamba malékite, ont préféré YouTube comme outil de " propagande » anti- ibadite. Les Ibadites, quant à eux, se sont concentrés sur l'usage de Facebook. On pourra ainsi mesurer le pouvoir incarné par les réseaux sociaux numériques et leurs acteurs dans la concurrence informationnelle faite aux médias traditionnels (presse, radio, télé...) et dans leur capacité à défier l'effacement des autorités publiques et à les obliger à prendre des décisions pour résoudre une crise qui aura duré trois ans.

7 De ce fait, comment l'usage des réseaux sociaux numériques a-t-il abouti au changement des opinions au sein de l'espace public afin d'influencer les décisions politiques des autorités algériennes? En d'autres termes, comment les réseaux sociaux numériques ont-ils accompagné déroulement de la crise à Ghardaïa? Dans quelle mesure les réseaux sociaux numériques ont-ils été un relais important pour éclairer l'opinion publique, et/ou la manipuler? Le choix adopté par Chaambas Malékites et Mozabites Ibadites des vecteurs d'information YouTube et Facebook est-il un facteur important dans la mobilisation sociale ? Nous tenterons de répondre à ces questions en nous appuyant sur les données dont nous disposons, alors même que ces événements sont récents et encore peu documentés. Cette étude cherche à donner un premier éclairage qui exige sans nul doute d'être complété et confronté à de futurs travaux.

8 Après avoir mobilisé quelques notions théoriques et explicité la méthodologie utilisée, nous allons appréhender les évènements de Ghardaïa dans la perspective des mouvements sociaux et de l'utilisation des médias numériques. Le retour sur la genèse de ces troubles nous permet de les placer dans un spectre analytique plus large qui fait appel à l'histoire, à la culture et à l'ethnographie. Nous analyserons par la suite quelques exemples de discours utilisés et véhiculés via les réseaux sociaux numériques par les icones de la contestation, ou ceux considérés comme tels notamment par la 
presse algérienne. Nous ferons appel également à la notion d'intelligence collective et la pertinence de son application sur le cas des évènements de Ghardaïa.

\section{Méthodologie et corpus de recherche}

Durant les émeutes de Ghardaïa, les réseaux sociaux numériques ont représenté un outil idéal pour la promotion des points de vue des deux parties (Ibadites Mozabites et Chaambas Malékites). Bien que l'étude porte plus précisément sur la période allant du 18 juin au 18 juillet 2015, soit celle de l'exacerbation de la crise, quelques retours en arrière étaient nécessaires.

10 Cette recherche est déterminée par deux principaux points : le premier est relatif à l'émergence des réseaux sociaux numériques comme vecteurs de messages allant tantôt dans le sens de l'amplification de la crise, tantôt dans celui de l'apaisement qui reflète une volonté de la collectivité nationale. Le second, porte, quant à lui, sur le choix des usagers pour tel ou tel type de vecteurs.

11 Pour comprendre ce phénomène, nous nous sommes focalisé sur deux types de réseaux sociaux numériques: YouTube et Facebook. Nous avons étudié les publications sur YouTube d'un cyber- activiste de 26 ans, Ahmed Seklab ${ }^{3}$. Ce dernier est présenté par la presse algérienne, aux côtés d'un autre protagoniste mozabite, le militant politique Kamal Eddine Fekhar ${ }^{4}$, comme des «leaders autoproclamés» de la contestation. Ces deux personnes sont présentées comme telles par plusieurs parties: d'abord leur entourage immédiat, la communauté à laquelle ils appartiennent ainsi que les médias. D'ailleurs, ces derniers ont largement considéré Seklab et Fekhar comme les incitateurs à la fitna à Ghardaïa.

12 En analysant les vidéos diffusées uniquement sur YouTube, et que nous avons consultées entre le 5 décembre et le 15 janvier 2015, nous constatons qu'Ahmed Seklab n'a pas hésité à utiliser un discours salafiste pour s'en prendre aux Mozabites. Bien que Seklab ait un compte Facebook, il a préféré utiliser YouTube dans le but de cibler le maximum d'audience et de pouvoir diffuser ses vidéos sur Facebook à partir de la plateforme YouTube. Il a bien compris que Facebook est un moyen de partage des vidéos postées sur YouTube.

13 Bien que postées entre le 14 mai 2014 et le 8 août 2015, quatre vidéos objets de l'analyse concernent les événements de Ghardaïa, tandis que deux sont des réquisitoires contre deux journalistes, avec pour toile de fond les émeutes dans la vallée du Mzab. Deux autres vidéos sont des réquisitoires contre le pouvoir algérien, deux autres sont relatives à son acte de défiance vis-à-vis de l'État (destruction de la carte d'identité et du diplôme du baccalauréat). Enfin, une vidéo fustige la ministre de l'Éducation nationale, alors qu'une autre est une réponse à un intellectuel qui a affiché son athéisme.

14 Une remarque mérite d'être soulevée : la chaîne YouTube de Seklab ${ }^{6}$ met en ligne neuf vidéos dont quatre seulement sont relatives aux questions politiques ; sur deux d'entreelles Seklab porte l'habit salafi des wahhabites saoudiens, les cinq autres traitent des aspects religieux et personnels. Les vidéos analysées proviennent donc d'autres comptes YouTube qui n'ont fait que répercuter les vidéos de Seklab, non disponibles sur sa chaîne. 

(département) qui s'étend sur $86105 \mathrm{~km}^{2}$ avec d'importantes distances entre ses limites nord et sud, soit $450 \mathrm{~km}$, et entre 200 et $250 \mathrm{~km}$ d'est en ouest ${ }^{8}$. Ghardaïa «dispose aujourd'hui d'une position remarquable au sein du Sahara algérien: relai sur le grand axe méridien Alger/Laghouat/Ghardaïa/Tamanrasset (la $R N n^{\circ} 1$ ), elle constitue le centre de gravité du territoire nord saharien. C'est à Ghardaïa que divergent les deux grandes 'boucles' des circuits sahariens, celle de l'Ouest vers Timimoun et la Saoura, celle de l'Est vers Ouargla et l'Oued Rhir " (Cote, 1998).

21 La population est estimée à 361570 habitants (recensement de 2007) ${ }^{9}$. Le dynamisme de la ville et de sa région a conduit à un taux d'accroissement annuel moyen de la population à hauteur de 2 alors qu'il est de 1,6 au niveau national (Oussedik, 2015). Deux groupes principaux constituent la majorité des habitants de la wilaya de Ghardaïa: les Mozabites, d'obédience ibadite et les Chaambas, sunnites d'obédience malékite. Les premiers se distinguent par leurs origines berbères, de la tribu des Zénètes et surtout par leur rite religieux. Ils sont Ibadites et constituent une des branches du Kharédjisme ${ }^{10}$, le plus ancien schisme de l'islam ${ }^{11}$, sorti des rangs des partisans de l'imam Ali ibn Abi Taleb, cousin et gendre du prophète Mohammed, dans 
les premiers moments de sa lutte avec Mu'awiya (futur fondateur de l'empire omeyyade). C'est cette caractéristique de mouvement marginal qui a imprégné l'évolution historique des Mozabites depuis la fondation des cités de la pentapole du Mzab aux Xe et XI e siècles (El-Atteuf 1011, Bou Noura 1048, Beni Isguen 1050, Ghardaïa 1053 et Malika 1124) (Golvin, 1994). Sur le plan organisationnel, c'est l'Assemblée des notables, Al-‘Azaba, qui gère la cité mozabite.

Les Chaambas, quant à eux, se réclament d'ascendance arabe « qui se revendiquent eux-mêmes comme descendants des Bani Sulaym ben Mansour de Médine (Hidjaz), qui sont une fédération de tribus» (Oussedik, 2015). Les Chaambas partagent avec la quasi-majorité des Algériens l'observation du rite sunnite malékite dans la pratique de la religion.

La conjonction des facteurs géographiques, historiques et humains font de cette région l'une des plus spécifiques d'Algérie. Mais faut-il pour autant parler de "communauté » et de "communautarisme» comme les médias ${ }^{12}$ l'ont évoqué pour expliquer les émeutes qui se sont produites en 1985, 1988, 1991, 2008, 2009, 2010, 2013, 2014 et 2015 dans cette région du Mzab ? Si celles des années 1980 et 1990 étaient confinées dans les litiges entre voisins de différentes origines, celles des années 2000 se caractérisent par l'importation de grilles de lecture moyen- orientales qui font des clivages entre doctrines religieuses et différents rites les principales sources d'affrontements intercommunautaires. Le problème est qu'en Algérie, il y a eu une stratification des clivages. Tantôt ethniques (Arabes/Berbères), tantôt identitaires (Chaambas/ Mozabites), ils deviennent très vite religieux (Malékites/Ibadites).

D'où le paradoxe du communautarisme en Algérie. Car « depuis quelques années, et en particulier avec ce qu'il est convenu d'appeler 'les événements de Ghardaïa', les notions d'ethnie, de minorité nationale ont fait leur irruption dans les débats politiques d'une Algérie dont l'existence se nourrissait d'un nationalisme exigeant. Dans ce contexte où l'idéologie nationaliste est nouée à une définition centralisée, à la française, de la Nation, cette situation nouvelle soulève des controverses souvent véhémentes » (Oussedik, 2015).

Les violences ont commencé le 19 décembre 2013 où les premiers affrontements ont enflammé la grande cité de la vallée du Mzab. Elles ont vu s'opposer tribus arabes (Chaambas) d'un côté et Mozabites, à quatre mois des élections présidentielles d'avril 2014.

Selon Abderrahmane Hadj Nacer, Mozabite et ancien gouverneur de la banque d'Algérie qui a signé plusieurs appels pour dénoncer les violences, «les conflits de voisinage ont toujours existé, mais l'État s'est décomposé, sans savoir construire sa légitimité» ${ }^{13}$. Ces dernières années, des heurts ont en effet éclaté à plusieurs reprises avec les Arabes malékites dominés par les Chaambas, mais ils n'avaient jamais atteint une telle intensité, causant d'importants dégâts matériels (incendie de biens) de part et d'autre. Dans le grand cimetière mozabite, un mausolée a été détruit, des tombes profanées. La plaque en marbre de l'Unesco qui rappelle que la vallée du Mzab figure sur la liste du Patrimoine mondial de l'humanité depuis 1982, a été vandalisée ${ }^{14}$.

Ainsi, comme il a été démontré plus haut, la crise de Ghardaïa, ou plutôt les crises cycliques que connaît cette région, ont des origines multiples d'ordres historique et économique, voire même politique, souvent éclipsées, soit par les médias, soit par les réseaux sociaux numériques, pour être présentées uniquement sous le spectre d'un conflit de type communautaire. La pratique discursive des deux parties conforte jusqu'à un certain point cette "dérive" communautariste, avant que l'intervention des 
autorités politico-militaires et les mécanismes de l'intelligence collective permettent de recadrer les discours dans le sens du vivre-ensemble au nom du slogan «Je suis Ghardaïa » ou encore celui de « Non à la fitna ».

\section{Mouvements sociaux et médias numériques}

Les événements de Ghardaïa sont aussi à appréhender dans la perspective des mouvements sociaux et d'utilisation des médias numériques. Tout mouvement social a des caractéristiques essentielles : une dimension collective, une définition des cibles et d'adversaires et des revendications. Autrement dit: les membres d'un mouvement social partagent un système de valeurs ou ont un projet en commun, se sentant liés par de la solidarité ou un sentiment d'appartenance; ils ont la capacité de mobiliser, de réunir un certain nombre de personnes pour des évènements ponctuels. Néanmoins, pour que le mouvement social existe, il faut de la continuité entre les moments forts (Lafrance, 2007).

Si l'expression "mouvement social» est ancienne, le concept sociologique lui, est récent, et prête à confusion. Deux acceptions principales opposent les sociologues entre eux, de façon tranchée. D'un côté, ceux pour qui le mouvement social est une action instrumentale correspondant à des fins politiques, une mobilisation des ressources, pour pénétrer au sein d'un système politique, s'y maintenir, y renforcer sa position. De l'autre côté, ceux pour qui le mouvement social est la signification la plus élevée d'une action collective à la fois contestataire et défensive, et visant au contrôle de l'historicité, c'est-à-dire à la maîtrise des orientations principales de la vie collective (Lafrance, 2007).

Les événements de Ghardaïa peuvent-ils être assimilés à une forme de mouvement social $?^{15}$ Considérant les précédentes définitions, les événements de 2014-2015 peuvent être considérés comme un mouvement social parce qu'ils présentaient la contestation d'une frange de la société algérienne contre une autre composante de la même société d'une part, et contre les autorités, accusées de laxisme, d'autre part. Aussi, ce mouvement se voulait une réaction défensive à ce que les Mozabites ont considéré comme étant une "agression" contre leur mode de vie et leurs biens. Enfin, ce mouvement social se voulait un "sursaut» qui devait rétablir les règles sociopolitiques tacites qui régissent la sociologie politique de l'Algérie indépendante dans le sens d'une plus grande intégration des Mozabites dans la dynamique nationale.

Ces événements ont révélé deux faits majeurs : les réseaux sociaux ont contribué à deux phases importantesdansleurdéroulement, l'exacerbationdeshainesentrelesdeuxprotagonistes Malékites et Ibadites, et l'apaisement. Ainsi que l'usage des réseaux sociaux numériques a été différemment approprié dans la mesure où les représentants des Malékites ont choisi principalement YouTube, tandis que ceux qui se réclament des Ibadites ont préféré Facebook. Un fait qui nécessite d'être interrogé : pourquoi cette spécificité dans le choix des vecteurs? C'est ce que nous allons essayer de comprendre à travers l'analyse des vidéos YouTube et des pages Facebook objets de notre recherche.

L'étude de cas de Ghardaïa est importante pour plusieurs raisons. D'une part, ce sont les controverses des Malékites et Ibadites qui ont alimenté les polémiques développées par les médias et autres réseaux sociaux numériques à l'occasion des émeutes de 2015. Chaque partie a utilisé les arguments communautaristes, chose inédite en Algérie, pour 
défendre ses positions. La blogosphère algérienne s'est fait l'écho de ces polémiques communautaristes, d'abord dans le sens de l'escalade, puis dans le sens contraire à savoir l'apaisement. D'autre part, le cas de Ghardaïa risque de se répéter à l'avenir, car pour certains représentants de ces communautés, les «représailles entre Malékites et Ibadites peuvent reprendre à n'importe quel moment ${ }^{16} \#$.

31 Au-delà de ces deux éléments, on note l'émergence du Salafisme takfiriste ${ }^{17}$, qui représente l'un des principaux résultats sanglants du printemps arabe, un phénomène très présent lors des évènements de Ghardaïa. D'ailleurs, l'une des principales caractéristiques des mouvements radicaux salafiste takfiriste est "l'utilisation des TIC. Par exemple, des sites rendant ainsi le jihad nonpas uniquement comme vérité globale, mais comme une vérité personnalisée » (Alterman, 2015).

L'usage des réseaux sociaux numériques a joué un grand rôle dans l'évolution de cette crise, voire dans sa gestion. Les vidéos publiées sur YouTube montrent que les cyberactivistes organisés en collectifs citoyens poursuivaient leur mobilisation pour relater leur vérité sur ces affrontements.

«Leur objectif est affiché : transmettre la vérité aux citoyens algériens lambda qui sont floutés par la propagande des autorités algériennes relayée par les médias classiques (télévision d'État, radios, agence de presse). Beaucoup d'activistes de Ghardaïa ainsi que des militants des droits de l'Homme considèrent que de nombreux faits sont occultés par ces médias ${ }^{18} »$. Toutefois les médias classiques et nouveaux sont critiqués en raison notamment de certaines notions qu'ils ont utilisées. Ainsi, pour l'ex-ministre du tourisme Abdelwahab Bakli (1992), originaire $\mathrm{du}$ Mzab, la presse ainsi que les réseaux sociaux ont utilisé «des concepts médiatiques impropres tels que 'conflit confessionnel' et 'différend entre Malékites et Ibadites' ${ }^{19}$ ».

Pour déconstruire le discours dominant relayé par les médias classiques notamment, internet a permis de construire des mobilisations informationnelles critiques de la production des médias, lesquelles ont évolué au fil des ans, sous le double effet de l'innovation technique et des logiques sociales (Granjon, 2014). Et l'utilisation d'internet dans le cas de Ghardaïa s'inscrit dans la même logique. En effet,

«internet facilite la publicisation des expériences, des interprétations et des revendications de groupes militants, des mouvements sociaux, mais aussi, plus récemment, de simples citoyens. Les espaces ainsi ouverts se construisent contre le formalisme de la mise en publicité des médias dominants, mais ils se défient également toujours davantage des formes d'encadrement militant de la parole. Avec plus ou moins de succès, ils participent au renouvellement des formats d'énonciation comme à celui des espaces d'interlocution et contribuent à faire évoluer les modalités de production de l'espace public » (Granjon, 2014).

Dans le cas de Ghardaïa, comment les réseaux sociaux numériques (Facebook, YouTube) ont-ils pu être non seulement un vecteur d'affrontement et un moyen d'expression d'hostilité, mais aussi de rapprochement et de conciliation et un outil encourageant le développement et la coexistence entre les communautés malékites et ibadites?

\section{YouTube pour les salafistes Chaambas, Facebook pour les Mozabites Ibadites}

En analysant les vidéos mentionnées dans le tableau $n^{\circ} 1$, et dontcertaines sonttoujours disponibles sur YouTube - la plupart ayant été supprimées pour incitation à la violence - on peut constater que le jeune Ahmed Seklab qui, selon les Mozabites, a contribué à 
l'amplification et à la dégradation de la situation entre Malékites et Mozabites, a distillé un discours contradictoire qui reflète son malaise sociétal. Il est important de noter que ce ne sont pas toutes ses vidéos qui ont été partagées sur sa page Facebook, bien qu'elle comptabilise 9930 abonnés. Cette dernière reflétait sa vie de jeune algérien qui postait ses vidéos personnelles à la plage, partageant son quotidien, chose qu'il ne pouvait pas faire sur YouTube. Ahmed Seklab voulait montrer son côté de jeune "salafiste modéré » qui nage même en short ${ }^{20}$.

Cependant, Ahmed Seklab a bien su cibler une importante masse. Le jeune étudiant en sociologie (Université d'Alger) et en théologie (Université de La Mecque), qui se voyait comme un modèle et une référence pour "une jeunesse perdue " selon lui, s'est autoproclamé opposant au gouvernement algérien. Il a utilisé un discours virulent pour se faire entendre et avoir une large audience. Le meilleur moyen a été de se révolter contre le gouvernement. Son acte contestataire fondateur a été de se filmer en train de brûler sa pièce d'identité et son attestation de baccalauréat dans la vidéo (C) (tableau $n^{\circ} 1$ ) avec 28441 vues, republiée dans les vidéos (D) et (E).

Son commentaire le plus virulent contre les autorités politiques a été posté dans la vidéo $(\mathrm{F})$ intitulée «Ahmed Seklab répond à l'athée Rachid Boudjedra ${ }^{21}$ ", publiée le 4 juin 2015, et qui a fait le buzz avec 127581 vues et 375 commentaires. Seklab distille un discours incohérent du point de vue islamiste. Tout en affirmant que Rachid Boudjedra est libre de croire ou pas, il se défend de vouloir appeler à son meurtre, car

« il ne faut pas succomber à la tentation de tuer les athées afin de ne pas donner une opportunité au pouvoir algérien qui n'attend que cette occasion pour réprimer les islamistes ». Pis, Seklab pousse très loin son "raisonnement » en s'en prenant directement au président algérien. Pour lui, «Bouteflika est un mécréant, car durant son ère, tous les excès ont été permis. C'est un pouvoir athée et mécréant. Son but est d'ostraciser les islamistes qualifiés d"extrémistes'« .

Tableau $n^{\circ}$ 1, Analyse des vidéos d'Ahmed Seklab sur YouTube (titre, minutage, nombre de vues)

\begin{tabular}{|l|l|l|l|}
\hline $\begin{array}{l}\text { Date } \\
\text { publication }\end{array}$ & Sujet & $\begin{array}{l}\text { La durée de } \\
\text { la vidéo }\end{array}$ & $\begin{array}{l}\text { Nombre de } \\
\text { vues }\end{array}$ \\
\hline $\begin{array}{l}\text { 09/08/2014 } \\
(\mathrm{A})^{22}\end{array}$ & $\begin{array}{l}\text { Seklab lance sa chaîne YouTube intitulée « la chaine } \\
\text { de Seklab } \\
\text { Abou Al Bara'23 » }\end{array}$ & $\begin{array}{l}196 \\
\text { abonnés }\end{array}$ \\
\hline $\begin{array}{l}\text { 04/08/2014 } \\
(\mathrm{B})^{24}\end{array}$ & Message d'un jeune au gouver- nement algérien & $10: 10$ & 17326 \\
\hline $\begin{array}{l}13 / 05 / 2015 \\
(\mathrm{C})^{25}\end{array}$ & $\begin{array}{l}\text { Contre le gouvernement algérien et les programmes } \\
\text { pédagogiques. Seklab brûle sa carte d'identité }\end{array}$ & $5: 53$ & 52041 \\
\hline $14 / 05 / 2015(\mathrm{D})$ & Seklab justifie son acte (brûler la carte d'identité) & $8: 42$ & 25198 \\
\hline $04 / 06 / 2015(\mathrm{E})$ & Seklab republie son acte (brûler sa carte d'identité) & $5: 03$ & 3647 \\
\hline $\begin{array}{l}\text { 04/06/2015 } \\
(\mathrm{F})^{26}\end{array}$ & Même contenu que la précé- dente & $5: 03$ & 127581 \\
\hline $02 / 07 / 2015(\mathrm{G})$ & Message au peuple algérien & $3: 56$ & 1810 \\
\hline
\end{tabular}




\begin{tabular}{|l|l|l|l|}
\hline $08 / 07 / 2015(\mathrm{H})$ & Un message aux jeunes qui ne vivent pas à Ghardaïa & $4: 07$ & 14270 \\
\hline $08 / 07 / 2015(\mathrm{I})$ & Contre les Ibadites et leurs notables « al-'Azaba » & $6: 47$ & 5528 \\
\hline $\begin{array}{l}18 / 07 / 2015 \\
(\mathrm{~J})^{27}\end{array}$ & Contre le journaliste Mohamed Tamalet & $12: 03$ & 6562 \\
\hline $20 / 07 / 2015(\mathrm{~K})$ & Contre les Ibadites et le journa- liste Ben Sedira & $13: 40$ & 6680 \\
\hline $21 / 07 / 2015(\mathrm{~L})$ & Contre la journaliste Leila Bouzidi & $11: 57$ & 21542 \\
\hline $\begin{array}{l}06 / 08 / 2015 \\
(\mathrm{M})\end{array}$ & Contre la ministre de l'Edu- cation & $8: 58$ & 1651 \\
\hline $25 / 11 / 2015(\mathrm{~N})$ & Message au gouvernement algérien & $8: 46$ & 24501 \\
\hline
\end{tabular}

Dans une autre vidéo $(\mathrm{G})$ intitulée «Nouveau: message d'Ahmed Seklab au peuple algérien », et publiée le 3 juillet 2015, soit au plus fort des événements de Ghardaïa, il se défait du discours anti-ibadite pour concentrer ses attaques contre le pouvoir algérie. Ainsi, selon lui, ce sont les intérêts de certains responsables à la tête de l'État qui sont derrière ces troubles.

«Le problème de Berriane ${ }^{28}$ est causé par Saïd Bouteflika [le frère du président] et les généraux Gaïd Salah et Toufik. Ils sont en train de réaliser un autre tracé pour la RN1 afin de contourner cette ville. Désormais, même si Malékites et Ibadites s'entretuent, le contournement de la RN1 permet de maintenir les affaires des puissants aux dépens du peuple».

Ainsi, Ahmed Seklab a clairement déclaré sur un ton vigoureux que l'État est l'unique responsable de la situation du pays, en précisant que le peuple algérien est uni par une forte fraternité et ne connaît pas de division. Cette dernière n'est, selon lui, que l'œuvre du seul gouvernement. Dans la même vidéo, il a affirmé n'avoir ni la nationalité algérienne, dont il n'est pas fier, ni son diplôme, car lors de la distribution des terres à Ghardaïa, on lui avait refusé sa demande comme le montre la vidéo (C ${ }^{29}$. Dans celle-ci publiée le 13 mai 2015 et intitulée « un jeune algérien brûle sa carte d'identité et son baccalauréat », l'auteur a réussi à faire le buzz avec 52041 vues et 151 commentaires. 
Graphe $N^{\circ} 1$, Analyse des vidéos d'Ahmed Seklab (''évolution du nombre de vues)

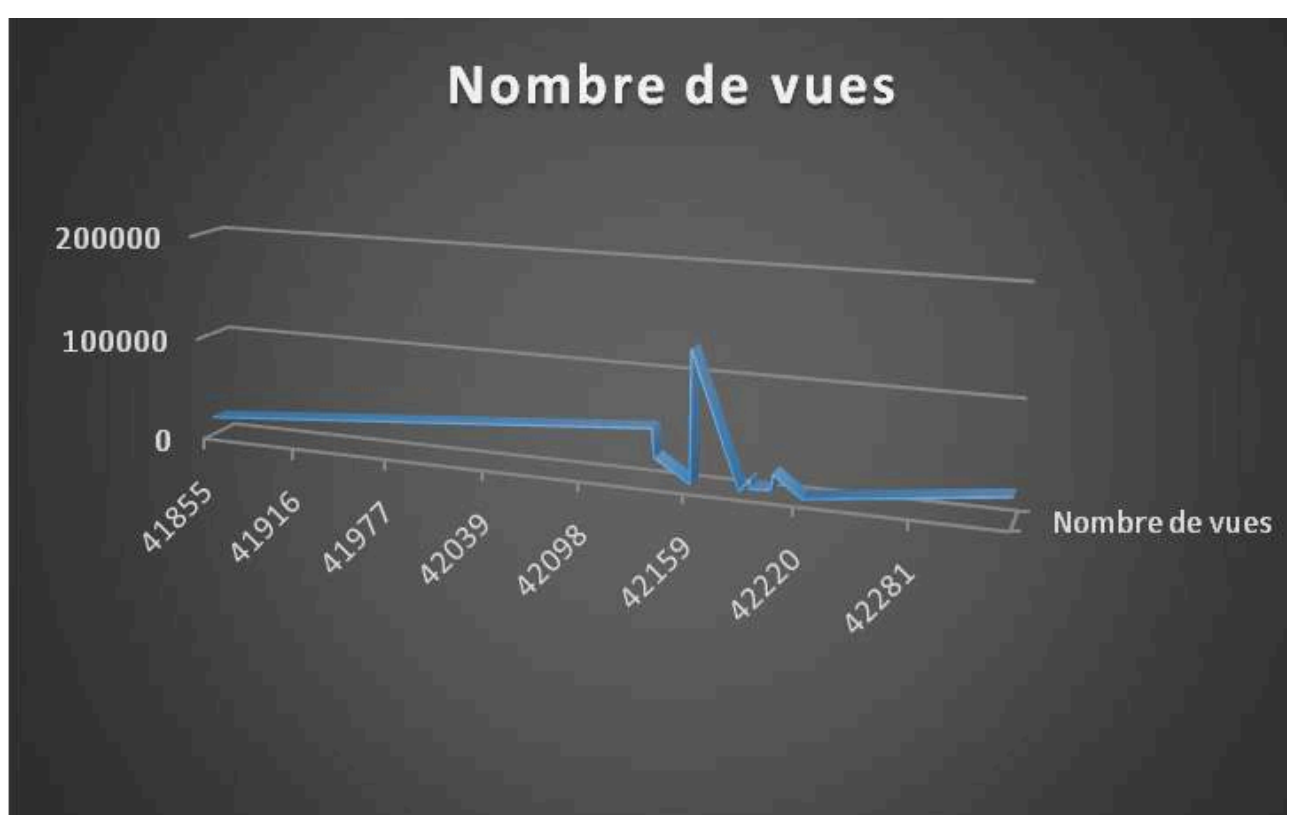

En analysant les vidéos publiées et le nombre de vues (graphe $n^{\circ} 1$ ), on constate que les vidéos relatives à la communauté mozabite ont été publiées durant le mois de Ramadan 2015 (juin- juillet 2015). Cette période correspond à la recrudescence de la violence dans plusieurs localités du Mzab, malgré l'importance de ce mois sacré pour les deux rites ibadite et malékite. Il est également clair qu'Ahmed Seklab a pu attirer une masse importante de visiteurs en publiant des vidéos qui fustigent le gouvernement, ou celles qui condamnent des personnalités connues comme la journaliste Leila Bouzidi. Cette présentatrice de télévision a qualifié, dans son émission d'Echourrouk TV (une chaîne du groupe de presse indépendant Chourouk) intitulée JT fou, Ahmed Seklab d'incitateur à la fitna à l'instar de Kamal Eddine Fekhar.

41 Ahmed Seklab s'est attaqué aux Ibadites car leur façon de vivre ne lui plaît pas. Il considère qu'il peut émettre cette opinion au nom de la liberté d'expression. Seklab pousse son ironie plus loin en s'interrogeant : « pourquoi le FIS $^{30}$ ne peut-il pas avoir les mêmes droits que les Mozabites? $»^{31}$. Le jeune activiste n'a pas hésité à exploiter l'importance du mois du Ramadan pour "s'acharner" contre les Mozabites et la spécificité de leur système social. Seklab, originaire de la wilaya de Ghardaïa, se présente comme le meilleur juge de sa région natale en accusant les Ibadites de ne pas s'impliquer dans la société, et de refuser tout échange avec les autres Algériens. La cause du problème, selon lui, c'est les «A'Azaba ", l'Assemblée des notables Mozabites qui gère le système social. Selon Ahmed Seklab, ce sont eux qui poussent les Mozabites aux affrontements pour inciter les Arabes à vendre leurs biens et permettre aux Mozabites de fonder leur État. Le pic du nombre de vues durant cette période, comme le montre le graphe, reflète non seulement la recrudescence de la violence, mais également l'intérêt porté au message anti-mozabite de Seklab. Ce dernier ne s'est pas uniquement présenté comme le défenseur des Malékites via les réseaux socionumériques, mais surtout comme l'une des victimes de ces affrontements.

Il affirme que les Ibadites sont rentrés chez lui par effraction pour le tuer; mais il a réussi à se sauver, lui et son père, en s'échappant par les toits. 
fait nécessite d'être interrogé : pourquoi Seklab (qui s'est autoproclamé leader des Arabes Chaambas) a-t-il choisi de se focaliser sur YouTube comme principal vecteur de sa propagande bien qu'il ait un compte Facebook ? Il est clair que cet activiste a préféré utiliser une stratégie de «communication» dans le ciblage de son audience. Celle-ci a consisté à promouvoir son message anti-ibadite via YouTube. Ses débuts de contestataire ont été marqués par des messages anti-gouvernementaux afin de s'assurer le maximum d'audience avant de faire basculer ses diatribes contre les Mozabites, ainsi l'audience était garantie, car appâtée par un précédent discours politique contre l'ordre établi. D'ailleurs, l'une des pistes explicatives de la stratégie de Seklab est celle relative au pari de cet activiste sur le nombre de partages sur Facebook. En faisant le buzz sur YouTube, une vidéo est aussitôt partagée sur Facebook et par un nombre important d'internautes. Et étant donné qu'il y a près de 8 millions d'Algériens qui disposent d'un compte Facebook, l'impact était assuré. De ce fait, Ahmed Seklab a, en théorie, utilisé uniquement YouTube, mais du point de vue opérationnel, il a mobilisé triplement ce vecteur, en diffusant directement ses vidéos et parce que cellesci ont été reprises par d'autres chaînes YouTube, diffusant ainsi son message. D'autre part, Seklab a visé, via YouTube, le nombre de partages sur Facebook.

Côté mozabite, cette communauté a cherché, de son côté, à faire entendre sa voix et ses mécontentements via les réseaux sociaux numériques.

«Des jeunes ont filmé sur leurs portables les affrontements et diffusé sur les réseaux sociaux des images d'une violence inouïe. Sur l'une d'elles, Baba Ismail Azzedine Ben Brahim, 22 ans, pris à partie par un groupe le 5 février 2014, est poignardé dans la ville de Ghardaïa. Sur d'autres, des manifestants arabes s'abritent derrière des policiers, preuve, selon leurs adversaires, d'un parti pris délibéré des autorités ${ }^{32} \%$.

Ainsi, les contacts entre les "sages ", les notables des deux communautés, ont été complètement rompus. Les Mozabites, contrairement aux Arabes Chaambas, ont diffusé deux messages à travers les vidéos en ligne : montrer les dégâts qu'ils subissent et la «connivence » de l'État avec les Arabes Chaambas Malékites contre les Ibadites ${ }^{33}$. Les principaux médias utilisés sont Ghardaïa TV avec 30514 abonnés, qui dispose d'une page Facebook et d'un compte YouTube, consacré spécialement à la diffusion des vidéos, et la chaîne Ghardaïa News ${ }^{34}$ avec 3028 abonnés.

En décryptant les messages véhiculés par Ghardaïa $T V$ et Ghardaïa News, qui sont à la base des pages d'information créées sur Facebook, on constate que les principaux objectifs consistent à dénoncer les actes des Malékites, plus précisément des Chaambas, et la connivence de l'État, qui contribue selon les Mozabites à protéger les Arabes et à opprimer les Ibadites. Certes, les Mozabites ont utilisé quelques expressions discriminatoires comme criminels ou Aa'rab qui veut dire bédouins ou, comme on trouve sur la page de couverture de Ghardaïa news, la phrase « les Mozabites sont vos maitres », et en considérant les Chaambas comme des Harkis ${ }^{35}$. Nous remarquons ici que le discours injurieux adopté par les deux parties se réfère à des notions qui relèvent de plusieurs sphères: ethnique (Chaambas, Mozabites), sociologique (maîtres, sujets), religieuse (salafistes, ibadites), et même historique (harkis). Mais l'objectif est également de faire un travail d'investigation pour identifier les responsables des dégâts.

Ghardaïa TV a diffusé un message pacifique des Arabes de Ouargla pour sensibiliser les communautés en utilisant des slogans comme "nous sommes tous Ghardaïa, non à la division, non à la fitna... ». Un autre message appelait à l'union de la nation, en partageant le communiqué du Conseil des notables du Ksar de Guerrara (Mozabites) le 18 juillet

Communication, technologies et développement, 3 | 2016 
2015. À relever également le message d'Omar Dadjari Chikh, notable du Mzab, daté du 17 juillet 2015, et destiné aux jeunes Algériens, qui les incite à l'union et à bannir la division. Un autre message a été diffusé par l'armée algérienne le 10 juillet 2015. Ces appels ont été publiés à une période bien précise car chargée de symbolique, l'approche de la fête de l'Aïd el fitr qui succède au Ramadan, une fête religieuse sacrée pour tous les Musulmans indépendamment de leurs rites.

Dans l'analyse de Ghardaïa TV et Ghardaïa News, on remarque que les Mozabites ont utilisé Facebook afin de toucher la diaspora mozabite surtout en Europe et la sensibiliser par rapport aux événements dont ils se disent victimes. Et en adoptant une méthode d'investigation, pendant les émeutes, ils ont voulu démontrer et déterminer les responsabilités de chaque partie, y compris celles des forces de l'ordre. En fin de compte, les Mozabites ont su transformer ces réseaux sociaux en vecteur pour propager la culture de la paix, après avoir diffusé les vidéos montrant l'horreur des émeutes. Ces chaînes ont également fait converger la volonté d'apaisement chez les Mozabites et chez les pouvoirs publics en diffusant l'appel de l'armée algérienne qui a coïncidé avec la période de la fête de l'Aïd el fitr.

\section{Peut-on parler d'intelligence collective à Ghardaïa ?}

Les réseaux sociaux numériques ont joué un rôle important dans l'évolution des événements. Jamais auparavant, ces réseaux n'ont eu un tel impact sur la société algérienne. Cependant, leur rôle est différemment apprécié. Par exemple, Nacer Laib, enseignant en Économie à l'Université de Paris 13, a précisé qu'ils «ont bien été des vecteurs de l'évolution de la crise $»^{36}$, contrairement à Abdellah Zekri, coordinateur du Collectif des Mozabites en Europe, qui trouve que «les réseaux sociaux n'ont fait que montrer la réalité $»^{37}$.

Dans le même contexte, des cyber-activistes citoyens, rassemblés sous Ghardaïa news ${ }^{38}$ ont poursuivi leur mobilisation pour obliger les autorités à agir contre les bandes armées qui s'attaquent aux citoyens mozabites en lançant des slogans racistes.

"Ahdath [événements de] Ghardaïa est aussi une autre communauté de citoyens journalistes qui active énormément en publiant des photos, témoignages et vidéos retraçant les violences dont est victime la population mozabite de la part des délinquants soutenus par des barons et narcotrafiquants mafieux et protégés indirectement par les services de sécurité ${ }^{39} »$.

51 Un point qui mérite d'être interrogé : quelle a été la position des autorités algériennes face à ces enjeux sociaux liées à l'usage numérique? D'abord, la banalisation! Le traitement des événements de Ghardaïa dans la rubrique des faits divers permet de dépolitiser une question éminemment politique. L'aggravation de la crise et la mobilisation des réseaux sociaux numériques ainsi que le rôle des télévisions privées ont poussé les autorités à s'impliquer davantage pour régler la crise, sachant que les autorités politiques manifestent une méfiance quasi instinctive vis-à-vis des réseaux sociaux numériques.

Les craintes politiques des autorités algériennes quant à l'usage des réseaux sociaux numériques traduisent leur perception de la force et de l'hégémonie de l'usage de ces réseaux qui deviennent de plus en plus un moyen de mobilisation et «d'engagement civique ( (Matos, 2008) pour la génération numérique (E-génération), pour laquelle la première source d'information passe par les réseaux sociaux numériques. Les chiffres 
parlent d'eux-mêmes : selon Ashraf Zeitoun, représentant de Facebook dans la région de MENA (Moyen-Orient et Afrique du Nord) « 7,7 millions d'Algériens utilisent leur compte Facebook, et ils sont aussi 1,7 million qui se connectent à Facebook via les smartphones. Aussi, le taux de pénétration de Facebook en Algérie est passé de12, $27 \%$ en mai 2013 à 18,1\% en mai $2014^{40} »$. Cette génération numérique s'est octroyé le droit de se détacher de la socialisation politique développée jusqu'à présent par les autorités du pays. Le mode opératoire de cette E-génération a généré une prise en charge quasi-autonome de ses revendications. En étudiant la crise de Ghardaïa, peut-on introduire, à ce niveau, le concept d'intelligence collective?

Cette dernière a pour finalité théorique, selon Pierre Lévy, de " comprendre de manière de plus en plus précise et opératoire le fonctionnement des groupes humains engagés dans une activité coopérative au moyen d'ordinateurs - ou de terminaux mobiles - en réseaux» (Lévy, 2003). Pour George Pór,

« l'intelligence collective est une manière de tracer un chemin à travers le chaos, le chaos qui représente le hautniveaudecomplexitédans lequel les individus sont plongés, c'est-à-dire des situations où le futur est non déterminé, imprévisible et surtout émergent » (Pór, 2008). Ainsi, il est possible d'affirmer que dans le cas de Ghardaïa, internet «qui rend possible un plus grand nombre d'interactions via l'environnement a augmenté la perception qu'a l'homme de l'intérêt collectif, jusqu'à l'identifier à l'intérêt individuel lui-même » (Laniau, 2009).

54 La transcendance quia caractérisé les réseaux sociaux numériques, les derniers jours des événements (début juillet 2015), a eu comme point de départ un slogan «Je suis Ghardaïa ", inspiré du slogan « Je suis Charlie » lancé après l'attaque contre le satirique français Charlie Hebdo en janvier 2015. Le fond en noir, tandis que "je suis » est en couleur blanche et « Ghardaïa » en couleur rouge, symbolisant le sang (fig. $n^{\circ} 1$ ).

Figure $n^{\circ} 1$ : Affiche « Je suis Ghardaïa »

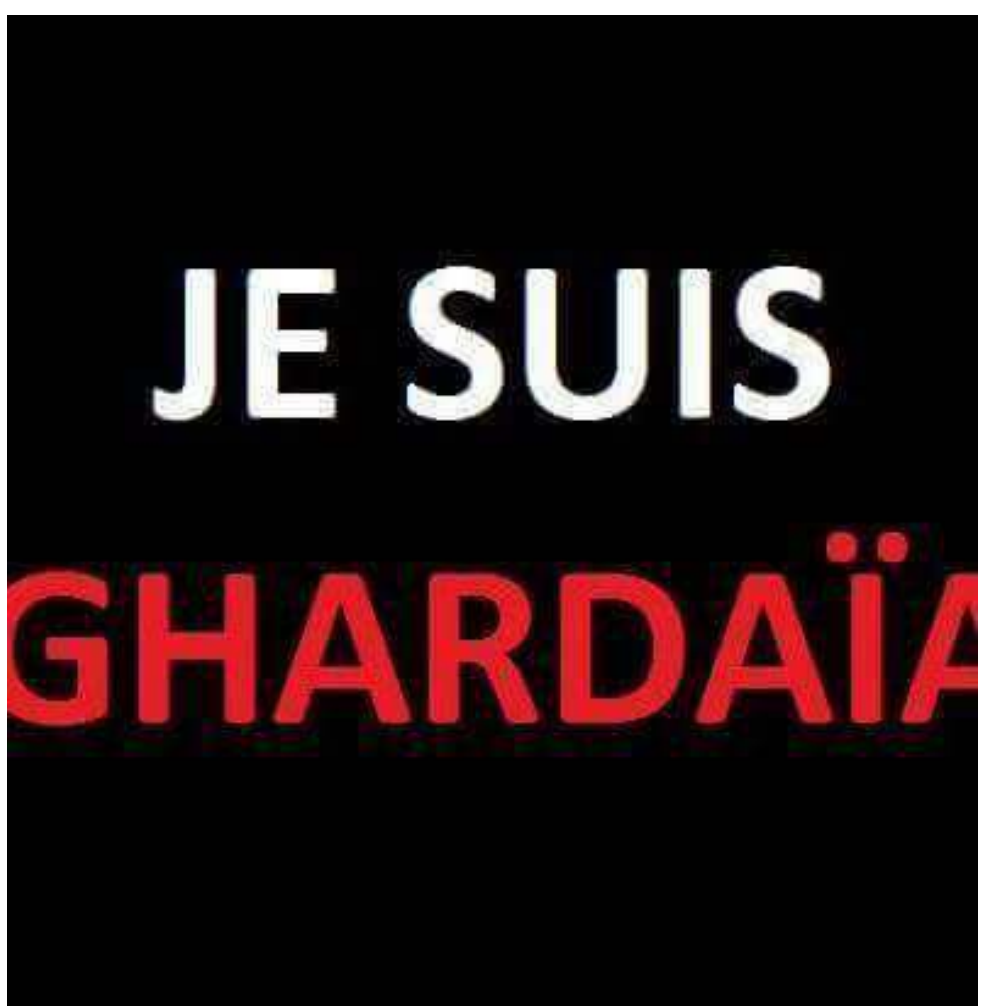


Le slogan écrit en français a été repris dans les langues arabe et tamazight (berbère) et reproduit sur une image de fond représentant une vue aérienne d'un ksar du Mzab. Pour le texte, le blanc et le rouge reviennent comme pour la précédente figure (fig. $\left.n^{\circ} 2\right)$.

Figure $n^{\circ} 2$ : Affiche « Je suis Ghardaïa » en arabe, en tamazight et en français

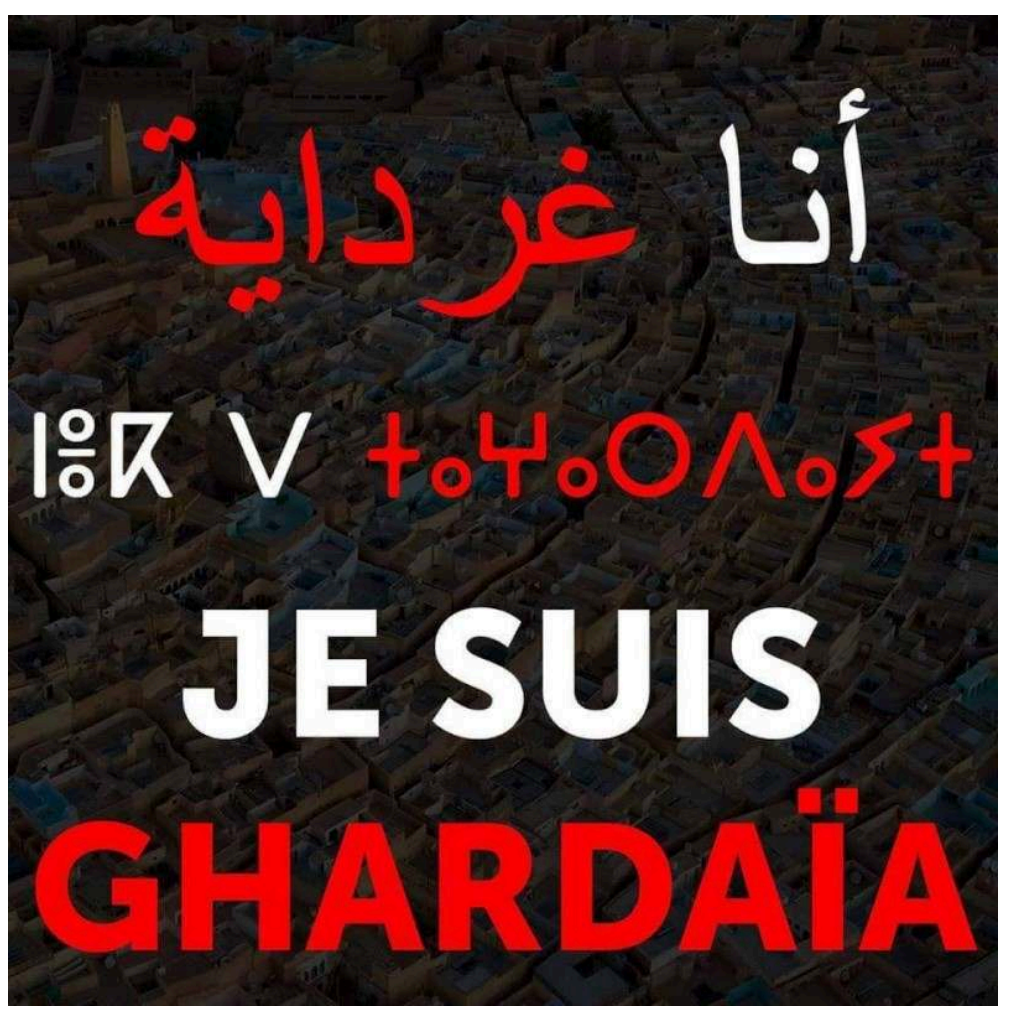

Un troisième slogan s'est également répandu sur les réseaux sociaux numériques, notamment Facebook, qui reprend la même logique, mais en donnant au slogan une plus grande signification symbolique, religieuse et politique. "Tous Ghardaïa, Non à la fitna (discorde)", précédé de hashtag, a été relayé par des milliers d'internautes algériens. Pour cette figure, la coloration du texte évolue : «\#Tous Ghardaïa " est en blanc, couleur de la paix, tandis que «\#Non à la fitna » est teinté en rouge, la couleur du sang (fig. $n^{\circ} 3$ ). 


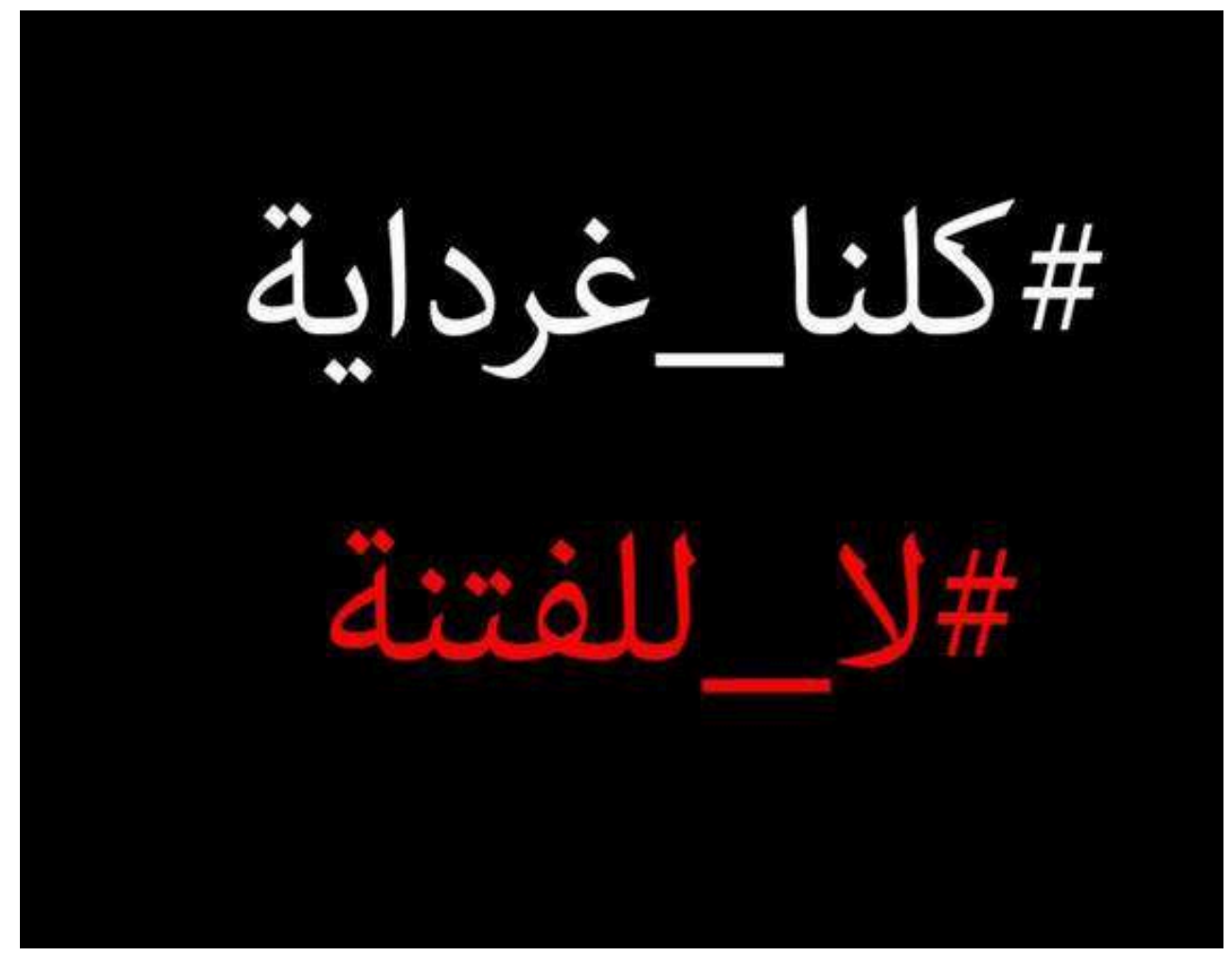

Figure $n^{\circ} 3$ : Affiche « \#Tous Ghardaïa, \#Non à la fitna (discorde) »

Image 200000090000321B000026E61B120FD5.wmf

57 En termes d'intelligence collective, une notion a été mobilisée, celle de «fitna [discorde] ». Toutes les parties (les protagonistes d'abord, le gouvernement, les médias et la blogosphère) ont repris cette notion devenue transversale. Est-ce pour autant que toutes les parties utilisent ce référent qui renvoie à l'histoire politique de l'islam des origines? Il serait intéressant de considérer la notion de

" fitna » comme un terme générique « dé-islamisé » à forte connotation sociopolitique plutôt qu'une notion éminemment religieuse.

59 Les événements de Ghardaiia ont démontré comment les réseaux sociaux numériques peuvent transformer une dynamique «négative» (polarisation des haines, exacerbation des sentiments, manipulation et propagande) en une dynamique " positive »(transcendance, retour au sentiment d'appartenance nationale, communion, etc.) sans que les pouvoirs politiques soient derrière cette transformation. C'est cette intelligence collective, basée sur l'efficacité et l'émergence qui a fait prévaloir l'intérêt collectif sur celui d'un groupe ou de quelques groupes, transcendant ainsi cet esprit de corps khaldounien, la 'Açabiya qui fait allégeance à un groupe selon les critères de filiations tribales ${ }^{41}$.

\section{Conclusion}

L'usage des réseaux sociaux numériques dans la crise de Ghardaïa nous amène à comprendre que ces outils ne sont pas uniquement un moyen d'incitation au désarroi et à la violence, mais une arme à double tranchant. En effet, l'exemple de Ghardaïa montre à la fois un usage qui amplifie les tensions dans la vallée du Mzab, mais également susceptible de les apaiser. 
61 À ce propos, il est nécessaire de déterminer l'importance de ces médias et leur pouvoir de créer une mobilisation sociale consciente, une intelligence collective autrement dit une e-opinion publique capable d'imposer la voix de la raison, de la sagesse et de la paix, et de régler ce que les politiques sont souvent incapables de résoudre. Certes, cette crise n'aurait pas pu se régler sans une volonté politique du gouvernement algérien, mais ce dernier n'aurait pas réagi sans la pression de la volonté sociale fortement incarnée par les réseaux sociaux numériques. Ceux-ci demeurent l'unique moyen de la société algérienne de se faire entendre. Ainsi, les crimes passés sous silence et l'impunité des auteurs de troubles ont été révélés publiquement, ce qui a mis les autorités algériennes dans une situation si délicate et si compromettante qu'elles ont été obligées d'imposer l'ordre public.

On soulignera que les réseaux sociaux numériques ont contribué au changement des opinions au sein de l'espace public à travers une opération en deux temps. D'abord, l'utilisation de ces médias sociaux, que sont YouTube et Facebook, pour les besoins de la propagande de chaque camp contre le camp adverse. Cette dualité YouTube/Facebook couplée aux protagonistes Malékites/Ibadites reproduit en quelque sorte une ligne de fracture et met en perspective un choix qui n'est pas fortuit, celui de l'utilisation, par chacun des deux camps d'un vecteur précis.

Il y a lieu de noter que l'activiste salafiste Ahmed Seklab a préféré utiliser YouTube afin de capter son audience grâce à un discours antigouvernemental d'abord pour la sensibiliser ensuite quant à la

64 «justesse » de sa position anti-ibadite, et d'utiliser enfin ces vidéos comme messages partagés par les utilisateurs de Facebook. Tandis que du côté mozabite, la prévalence de Facebook est soulignée dans la propagation et la diffusion des messages et des informations.

Le second temps consiste, quant à lui, en l'utilisation de ces réseaux comme un moyen pour appeler au retour de la paix et l'importance de la coexistence. Ces appels émanent autant des sages malékites et mozabites que des autorités politiques et militaires du pays. Ainsi, on est passé dans le déroulé de la crise de la manipulation de l'opinion publique, locale et nationale, via les réseaux sociaux numériques, à la construction d'un espace public solidaire, par le biais de l'intelligence collective, qui utilise ces mêmes réseaux pour promouvoir la paix sociale.

Quels enseignements pouvons-nous tirer des évènements de Ghardaïa en termes de mouvements sociaux et d'intelligence collective? Ces émeutes, émanation d'un mouvement social dans une région particulière de l'Algérie, ont un rapport à l'intelligence collective qui se développe dans un contexte, soit une mobilisation citoyenne dans un contexte précis (sur fond religieux, dans un premier lieu du moins).

67 Aussi, nous pouvons constater la recherche d'un dosage intelligent entre des pratiques et des expressions de mobilisation modernes, par exemple «Je suis Ghardaïa » inspiré de "Je suis Charlie", et des considérations liées au contexte social local. Ainsi, l'utilisation de la notion de " fitna " produit du sens dans la mesure où elle parle à la population, et de ce fait, vient en appui aux appels à la paix. Nous mesurons ici l'impact du contexte social sur la mobilisation dans la mesure où une notion religieuse est mise à contribution dans un ensemble de pratiques et d'expressions citoyennes donnant lieu à une véritable intelligence collective qui a contribué à 
68

\section{BIBLIOGRAPHIE}

BARDIN, Laurence. "L'analyse de contenu et de la forme des communications ", in MOSCOVICI Serge et BUSCHINI Fabrice (dir.), Les méthodes des sciences humaines, Paris : PUF, 2003.

COTE, Marc. « Ghardaïa ». Encyclopédie Berbère, Volume XX, Paris : Edisud, 1998.

DURAND Caroline, «L'art de faire émerger l'intelligence collective comme processus de changement émergent ». Mémoire présenté comme exigence partielle de la maîtrise en communication, Université du Québec, décembre 2011.

GOLVIN, Lucien. «Cités mozabites », Encyclopédie Berbère, Volume XIII. Paris : Edisud, 1994.

GRANJON, Fabien. « Médias dominants, mouvements sociaux et mobilisations informationnelles ", in Michel Pigenet et al., Histoire des mouvements sociaux en France, Paris : La Découverte, 2014.

LAFRANCE, Jean-Paul. « Les médias face à la communication sociale : le paradoxe canadien », Hermès, La Revue, 2007/2.

LANIAU, Jérôme. « Vers une nouvelle forme d'intelligence collective ? ", Empan, 2009/4 (n 76$)$. LEVY, Pierre. « Le jeu de l'intelligence collective », Sociétés, 2003/1, Nº 79.

MATOS, Heloiza. «Engagement civique et participation politique : controverses sur les TICs et le déclin du capital social », Les Enjeux de l'information et de la communication, $\mathrm{n}^{\circ}$ 1, 2008.

OUSSEDIK, Fatima. « Les émeutes de Ghardaïa. L'Algérie, une société en guerre contre ellemême ", Revue NAQD, n³2, automne 2015.

PÓR, George. «Collective Intelligence and Collective Leadership ; Twin Paths to Beyond Chaos », All Sprouts Content, Paper 207, 2008.

SAYARH, Nada. «La netnographie : mise en application d'une méthode d'investigation des communautés virtuelles représentant un intérêt pour l'étude des sujets sensibles ", Recherches Qualitatives, vol. 32 (2), 2013.

Webographie 
AGERON, Charles Robert. « Mozabites », Encyclopædia Universalis [en ligne], consulté le 11 février 2016. http://www.universalis.fr/encyclopedie/mozabites/

ALGERIE-FOCUS (redaction avec APS). « Violence à Ghardaïa : Un ex-ministre mozabite accuse "les barons de la drogue et de la contrebande" » (03/02/2014). http://www.algerie-focus.com/ 2014/02/violence-a-ghardaia-un-ex-ministre-mozabite-accuse-les-barons-de-la-drogue-et-de-lacontrebande/. Consulté le 14/12/2015.

ALGERIE PRESSE SERVICE, «Incidents de Ghardaïa : les interpellations des personnes présumées impliquées conformes à la loi (Procureur)» (13/07/2015). https://www.algerie360.com/ incidents-de-ghardaia-les-interpellations-des-personnes-presumees-impliquees-conformes-a-laloi-procureur/.

ALTERMAN, Jon B. « Religious radicalism after the Arab Uprisings », https://www.csis.org/ analysis/religious-radicalism-after-arab-uprisings. Consulté le 07/12/2015.

K. K. « Ghardaïa : les habitants crient au complot ». Liberté (29/01/2014) https://www.libertealgerie.com/actualite/ghardaia-les-habitants-crient-au-complot-200629.

LYES, Sonia. « Kamel Eddine Fekhar condamné à un an de prison ferme » (26/10/2015). https:// www.dzairnews.com/articles/tsa-kamel-eddine-fekhar-condamne-a-un-an-de-prison-ferme

MANDRAUD, Isabelle. « En Algérie, Ghardaïa enflammée par les violences communautaires ». Le Monde Afrique (19/02/2014). http://www.lemonde.fr/afrique/article/2014/02/19/en-algerieghardaia-enflammee-par-les-violences-communautaires_4369303_3212.html.

Ministère de l'Intérieur et des Collectivités locales (Algérie), Portail officiel, http:// www.interieur.gov.dz/Dynamics/frmItem.aspx?html=35\&s=26.

SEMMAR, Abdou. Les cyber-activistes algériens dévoilent un crime raciste à Ghardaïa (06/02/2014). https://fr.globalvoices.org/2014/02/06/161577/.

SEMMAR, Abdou. « 7,7 millions d'Algériens ont un compte Facebook et 3,6 millions s'y connectent chaque jour » (15/02/2015). https://www.algerie-focus.com/2015/02/77-millionsdalgeriens-ont-un-compte-facebook-et-36-millions-sy-connectent-chaque-jour/

SOUKOU, Nouar. « Le terrain miné du conflit confessionnel à Ghardaïa. Des appels lancés par les notables de la région pour sauver la coexistence entre Arabes et Mozabites ». El-Khabar (18/12/2013) in http://www.djazairess.com/elkhabar/374238.

Wilaya de Ghardaïa. Portail officiel, http://wilayadeghardaia.dz

Pages Facebook

Page Ghardaïa TV https://www.facebohttps://www.facebook.com/a7dath.ghardaia/? fref=ts30514 30514 abonnés (consulté le 27/01/2016), 30126 abonnés le 16/05/2016.

Page Ghardaïa News https://www.facebook.com/mzabghardaia/?fref=ts30283028 abonnés (consulté le 27/01/2016), 3157 abonnées le 16/05/2016.

Chaîne YouTube

Chaîne d'Ahmed Seklab Abou Al Bara' https://www.youtube.com/channel/ UCdjRVGsw5WFutpntGqU9SFQ, 196 abonnés.

Vidéographie YouTube

SEKLAB, Ahmed. « Nage professionnelle ». Ajouté le 30/07/2014, https://www.youtube.com/ watch? $=\mathrm{dECUD} 9 \mathrm{XRZdU}, 181$ vues. 
SEKLAB, Ahmed. « Message d'un jeune au gouvernement algérien ». Ajouté le 04/08/2014, https://www.youtube.com/watch?v=2MXJD35U3SY, 17328 vues.

SEKLAB, Ahmed. « Message au gouvernement algérien après l'exclusion d'Ahmed Seklab des rangs de l'université ». Ajouté le 25/11/2014, https://www.youtube.com/watch?v=uF--_7YF-xs, 24754 vues.

SEKLAB, Ahmed. «Jeune algérien qui brule sa carte nationale et son diplôme de baccalauréat ». Ajouté le 13/05/2015, https://www.youtube.com/watch?v=82EmPokZHjM, 52208 vues.

SEKLAB, Ahmed. «L'étudient Ahmed Seklab explique quelques faits sur son acte (bruler sa carte d'identité) ». Ajouté le 14/05/2015, https://www.youtube.com/watch?v=qOHzmLQZIBQ 26493 vues.

SEKLAB, Ahmed. «Ahmed Seklab répond à l'athée Rachid Boudjedra ». Ajouté le 04/06/2015, https://www.youtube.com/watch?v=AhaATr6VAsc, 127710 vues.

SEKLAB, Ahmed. « Réponse de Seklab au journaliste futile (sic) Mohamed Tamalt ». Ajouté le 18/07/2015, https://www.youtube.com/watch?v=5zEduMoCi8o, 7949 vues.

Entretiens téléphoniques avec l'auteur

LAIB Nacer, enseignant en économie à l'Université Paris 13, et membre du Collectif Mozabite en Europe, 02/02/2015.

ZEKRI Abdallah, président de l'Observatoire de l'islamophobie, membre de la direction de la Fédération de la Grande Mosquée de Paris, coordinateur du Collectif des Mozabites en Europe, 27/01/2016.

\section{NOTES}

1. Ces amendements concernent, notamment, la limitation des mandats, la dépénalisation du délit de presse et la parité hommes-femmes.

2. Les Chaambas, tribus d'origine arabe dont les membres relèvent de plusieurs wilayas (départements) notamment Ghardaïa et Ouargla. Du point de vue religieux, les Chaambas sont, à l'instar de la majorité des Algériens, des sunnites d'obédience malékite.

3. Ahmed Seklab, jeune Algérien originaire de la wilaya de Ghardaïa appartenant aux tribus arabes Chaambas. Son père a été tué lors des émeutes de 2014. De tendance salafiste, il a contribué par ses vidéos postées sur YouTube à attiser la haine contre les Mozabites car, nantis économiquement et financièrement, selon lui, contrairement aux Arabes Chaambas. Seklab a été arrêté par les autorités en août 2015. Cf. http:// algeriesolidaire.net/ghardaia-arrestation-dahmed-seglab/

4. Kamal Eddine Fekhar, militant des droits de l'Homme et responsable de la fédération des Frontdesforcessocialistes(FFS),leadermozabite proche des thèses autonomistes du Mouvement pour l'autonomie de la Kabylie (MAK) qui présente les Mozabites et les Kabyles comme des minorités persécutées par les autorités. Accusé d'être l'un des principaux instigateurs, côté mozabite, des émeutes de Ghardaïa, il a été arrêté et condamné à un an de prison ferme pour plusieurs chefs d'inculpation. Voir : https:// www.algerie360.com/incidents-de-ghardaia-les-interpellations-des-personnespresumees-impliquees-conformes-a-la-loi-procureur et https://www.dzairnews.com/ articles/tsa-kamel-eddine-fekhar-condamne-a-un-an-de-prison-ferme 
5. Fitna un mot arabe qui signifie discorde entre deux communautés, parties, groupes de populations, quels que soient les motifs. Cette notion à forte connotation religieuse désigne les guerres fratricides entre les compagnons du prophète Mohamed notamment après l'assassinat du 3e calife « bien guidé » Othman Ibn 'Affan, l'avènement du calife Ali Ibn Abi Taleb et la contestation de son pouvoir par M'uawiya Ibn Abi Sofiane, le fondateur de la dynastie Omeyyade à Damas. Cf, Hicham Djaït, $L a$ Grande Discorde : religion et politique dans l'islam des origines. Paris : Gallimard, 1989. En Algérie, où le pluralisme religieux et politique fait défaut, le moindre problème peut être qualifié de fitna. Ainsi, l'usage excessif de cette notion finit par la vider de son contenu.

6. https://www.youtube.com/channel/UCdjRVGsw5WFutpntGqU9SFQ/videos.

7. Cet aspect cyclique sera développé plus loin.

8. Portail de la wilaya de Ghardaïa, http://algeriesolidaire.net/ghardaia-arrestationdahmed-seglab.dz/

9. Portail du ministère algérien de l'Intérieur et des Collectivités locales. http:// www.interieur.gov.dz/index.php/fr/.

10. Le kharédjisme est donc, à la base, une doctrine qui ne fait pas partie du sunnisme, et qui a été combattue par le chiisme.

11. Cf, Charles Robert Ageron, « Mozabites », Encyclopædia Universalis [en ligne], consulté le 11 février 2016. http://www.universalis.fr/encyclopedie/mozabites/

12. Voir par exemple : Soukou, Nouar. « Le terrain miné du conflit confessionnel à Ghardaïa. Des appels lancés par les notables de la région pour sauver la coexistence entre Arabes et Mozabites ». El-Khabar (18/12/2013) in http://www.djazairess.com/ elkhabar/374238

13. Isabelle Mandraud, « En Algérie, Ghardaïa enflammée par les violences communautaires », Le Monde Afrique, 19/02/2014, https://www.lemonde.fr/afrique/ article/2014/02/19/en-algerie-ghardaia-enflammee-par-les-violencescommunautaires_4369303_3212.html.

14. Ibid

15. Pour plus de détail sur la notion de mouvements sociaux au Maghreb, voir Didier Le Saout et Marguerite Rollinde (dir.), Ementes et mouvements sociaux au Maghreb. Perspective comparée. Paris : Karthala, 1999.

16. Entretien téléphonique, le $02 / 02 / 2015$, avec Nacer Laib, enseignant en économie à Paris 13, et membre du collectif Mozabites en Europe.

17. Le takfirisme se réclame de l'ultra-rigorisme et tend à apostasier quiconque n'adhère pas à ses postulats. Le takfirisme est souvent associé au salafisme et au wahhabisme.

18. Sur ce point notamment, voir Abdou Semmar, « Les cyber-activistes algériens dévoilent un crime raciste à Ghardaïa ». In globalevoices.com, consulté le 06/02/2014. 19. "Violence à Ghardaïa : Un ex-ministre mozabite accuse "les barons de la drogue et de la contrebande" ». In http://www.algerie-focus.com/2014/02/violence-a-ghardaiaun-ex-ministre-mozabite-accuse-les-barons-de-la-drogue-et-de-la-contrebande/ consulté le 14/12/2015.

20. La page Facebook de Seklab ayant été supprimée, une vidéo le montrant se baignant à la piscine est toujours disponible sur sa chaîne Youtube sur le lien https:// www.youtube.com/watch?v=dECUD9XRZdU.

21. Rachid Boudjedra, écrivain et essayiste algérien, auteur notamment de L'escargot entêté (Denoël, 1977) et Le Printemps (Grasset, 2014), a déclaré publiquement assumer 
son athéisme lors d'une émission de télévision diffusée sur Echourrouk TV, le 3 juin 2015.

22. https://www.youtube.com/channel/UCdjRVGsw5WFutpntGqU9SFQ.

23. Abou Al Bara' surnom pris par Seklab fait référence à un compagnon du prophète Mohamed. Les salafistes ont pour habitude de prendre comme surnoms des noms de compagnons ayant vécus l'épopée prophétique.

24. https://www.youtube.com/watch?v=2MXJD35U3SY.

25. https://www.youtube.com/watch?v=82EmPokZHjM.

26. https://www.youtube.com/watch?v=AhaATr6VAsc.

27. https://www.youtube.com/watch?v=5zEduMoCi8o.

28. Berriane est une commune de la wilaya de Ghardaïa, sur le tracé de la RN1 qui relie Alger au sud du pays. Elle est composée majoritairement de population d'origine chaambas, qui côtoie une population d'origine mozabite.

29. https://www.youtube.com/watch?v=82EmPokZHjM.

30. Le Front Islamique du Salut, créé en février 1989, dissous en mars 1992. Ce parti, qui a gagné les élections municipales de juin 1990, et le premier tour des législatives de décembre 1991, a basculé dans la violence après l'interruption du processus électoral en janvier 1992. Cette violence a duré jusqu'en 1999 avec 200000 morts.

31. https://www.youtube.com/watch?v=YMxrTRi_dPs.

32. Isabelle Mandraud, op. cit.

33. https://www.facebook.com/a7dath.ghardaia/?fref=ts. Voir aussi, http://

www.liberte-algerie.com/actualite/ghardaia-les-habitants-crient-au-complot-200629/print/1.

34. https://www.facebook.com/mzabghardaia/?fref=ts3028.

35. Traître, appellation utilisée en Algérie pour désigner tout Algérien ayant servi le colonialisme français.

36. Entretien téléphonique avec Nacer Laib, Op. cit.

37. Entretien téléphonique avec Abdallah Zekri, de l'observatoire de l'islamophobie, membre de la direction de la Fédération de la Grande Mosquée de Paris, le coordinateur du Collectif des Mozabites en Europe (27/01/2016).

38. https://www.facebook.com/mzabghardaia/?fref=ts.

39. Abdou Semmar, Op. cit.

40. Abdou Semmar, «7,7 millions d'Algériens ont un compte Facebook et 3,6 millions s'y connectent chaque jour» (15/02/2015). http://www.algerie-focus. com/2015/02/77millions-dalgeriens- ont-un-compte-facebook-et-36-millions- sy-connectent-chaquejour/

41. Pour la pensée khaldounienne, voir Abderrahman Ibn Khaldoun, Discours sur l'Histoire Universelle (Al-Muqaddima). Traduction nouvelle, préface et notes par Vincent Monteil. 3e édition, Arles : Acte Sud, 1997. 


\section{RÉSUMÉS}

Le présent article se propose de jeter la lumière sur l'usage des réseaux sociaux numériques dans la crise de Ghardaïa en Algérie entre 2014 et 2015, et de comprendre les ressorts du discours utilisé tantôt pour attiser l'affrontement, tantôt pour une posture plus conciliante. Pour ce faire, ce travail de recherche mobilise deux notions clés: les mouvements sociaux et l'intelligence collective.

Cette étude de cas a pour objectif d'éclairer une pratique encore peu répandue en Algérie, à savoir la mobilisation sociale et politique via les réseaux sociaux numériques. Elle s'appuie sur quelques exemples de discours utilisés et véhiculés via ces réseaux par les icones de la contestation, ou ceux considérés comme tels. Nous ferons appel également à la notion d'intelligence collective et la pertinence de son application sur le cas des évènements de Ghardaïa. Cette crise a confirmé, s'il en est, cette conscience nouvelle quant aux possibilités que présente l'utilisation de YouTube et de Facebook comme vecteurs à même d'influencer la vie de la collectivité nationale. Les événements de Ghardaïa ont démontré comment les réseaux sociaux numériques peuvent transformer une dynamique «négative» (polarisation des haines, exacerbation des sentiments, manipulation et propagande) en une dynamique "positive " (transcendance, retour au sentiment d'appartenance nationale, communion, etc.) sans que les pouvoirs politiques soient derrière cette transformation. C'est cette intelligence collective, basée sur l'efficacité et l'émergence qui a fait prévaloir l'intérêt collectif sur celui d'un groupe ou de quelques groupes.

This article is based on the use of social networks in the events of Ghardaia in Algeria between 2014 and 2015, and on understanding the deep of the speech, sometimes used to stir up confrontation, sometimes to a more conciliatory posture. To do this research mobilizes two key concepts : social movements and collective intelligence.

This research tries to clarify the practice that is still uncommon in Algeria, which is : the social and political mobilization through social networks. This study is based on a few examples of speech used via these networks, specially, by the icons of the conflict between Ibadites and Malikites. We will also use the concept of collective intelligence and relevance of its application in the case of events of Ghardaia. Indeed, these events confirmed, how the use of YouTube and Facebook as a mean of information can influence the life of the Algerian community.

Ghardaia events have shown how digital social networks can turn a "negative " dynamic (polarization of hatred, exacerbation of feelings, manipulation and propaganda) in a " positive " dynamic (transcendence back to the sense of national belonging, communion, etc.). This collective intelligence, based on efficiency and emergence, made the collective interest prevail over that of a group or several groups.

\section{INDEX}

Keywords : social network, youtube, facebook, algeria, ghardaïa, collective intelligence.

Mots-clés : réseaux sociaux numériques, youtube, facebook, algérie, ghardaïa, intelligence collective 
AUTEUR

RIMA ROUIBI

École Nationale Supérieure de Journalisme et des Sciences de l'Information (Alger), Laboratoire MUSC (ENSJSI-Alger). 